\title{
Terrorismus ist keine Krankheit
}

\author{
Die Regierung plant Maßnahmenvollzug für \\ potenzielle Gefährder und Terroristen. Harald David
}

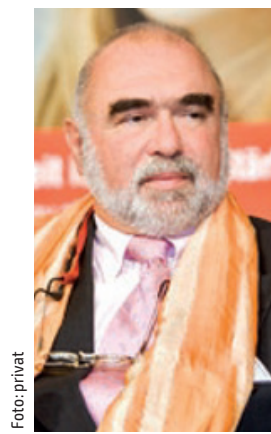

MEDIZINALRAT DR. HARALD DAVID Psychiater, Wien david.psych.log aaon.at
Das Zitat „Wer Visionen hat, soll zum Arzt gehen“ wird zwar Vranitzky zugeschrieben, soll er aber nie gesagt haben. Der Satz „Wenn geistig abnorme Rechtsbrecher lebenslang weggesperrt werden können, wenn eine Gefahr von ihnen ausgeht, kann auch ein Terrorist lebenslang weggesperrt werden" hätte von Kurz nie gesagt werden sollen; hat er aber gesagt. Er sprach auch den Wunsch aus, vorbeugende Maßnahmen gegen zukünftige GefährderInnen einzuführen.

Schon 1973 kommt der Wiener Psychoanalytiker Friedrich Hacker in seinem Werk Terror in seiner Definition nicht einmal in die Nähe von Krankheit. Er schreibt: „Terror ist die Verwendung des Herrschaftsinstruments der Einschüchterung durch die Mächtigen, Terrorismus die Nachahmung und Praxis von Terrormethoden durch die (noch) Machtlosen, Verachteten und Verzweifelten, die glauben, auf keine andere Weise ernst und für voll genommen zu werden."

Es gibt derzeit zwei schwere Missverständnisse. Das erste ist eines über das Wesen des Maßnahmenvollzugs. Sein Ziel ist die Anleitung von Menschen, die aufgrund einer psychischen Krankheit oder Störung (im Gesetzestext leider immer noch „geistige oder seelische Abartigkeit höheren Grades“) dazu neigen, Probleme auf eine höchst gefährdende Weise zu lösen, damit sie im dafür nötigen Zeitraum andere Strategien lernen und festigen. Für die Beurteilung des Erfolgs werden sichere Freiräume zur Erprobung und Umsetzung benötigt. Das Ziel ist also Veränderung und Eigenverantwortung.

Das zweite Missverständnis liegt in der Idee des „Wegsperrens“. Weg von wem oder wovon? Da wir diese Menschen nicht ins Weltall verfrachten können, müssen und wollen wir eben damit leben, dass sie auch wieder Kontakt mit Teilen der Gesellschaft haben oder sogar voll wieder in diese zurückkehren.

Was nun Terroristen betrifft, kann die Strategie des „Wegsperrens“ nur zu weiteren Problemen führen, weil diese ohnehin oft mit dem Gefühl leben, nicht Teil dieser Gesellschaft zu sein und auch keine adäquate Möglichkeit zu finden hineinzukommen. Sie haben oft eher den Eindruck, von vornherein nicht willkommen oder akzeptiert zu sein.
Wegsperren - Isolieren - Ausgrenzen sind also Machtinstrumente, die nur mehr vom Selben hervorrufen. Das heißt nicht, dass die Wege zur Veränderung nicht sehr klar beschrieben und konsequent beobachtet werden, dass Abweichungen nicht unverzüglich als solche bezeichnet und korrigiert werden sollen. Das heißt aber, dass beim Umgang mit Terroristen genauso wie mit Maßnahmenpatienten immer auch Bewegung und Veränderung möglich sein muss.

Norbert Nedopil, Psychiater und gesuchter Gutachter, warnt in seinem Buch Jeder Mensch hat seinen Abgrund vor den Sekundärschäden von Terroranschlägen durch das Entstehen von Misstrauen, Spaltung in der Gesellschaft und Vermeidungsverhalten. Ausgrenzung würde das fördern.

Für mögliche Gefährder sieht das Gesetz die Strafbarkeit der Vorbereitung rechtswidriger Handlungen bzw. der Bildung von Vereinigungen zur Durchführung von solchen vor. Außerdem dürfen nur begangene Rechtsverstöße - und die Vorbereitung rechtswidriger Handlungen zählen eben dazu - geahndet werden. Das Nachdenken darüber ist nicht strafbar darf es auch nicht sein, weil sonst keine Möglichkeit bestünde, sich dagegen zu entscheiden.

\section{Wegsperren allein geht gar nicht}

Reinhard Haller schreibt in seinem Buch Das Böse, dass es nach allen Untersuchungen keine Hinweise gibt, dass eine typische Terrorpsyche existiert und dass Terroristen wirklich psychisch krank sind. Terrorismus ist meist ein Eskalationsprozess, erzeugt durch Gruppenideologie und die gruppenbezogene Legitimation. Damit ist die Idee des gruppenweisen Wegsperrens von vornherein absurd, weil dies zur Schaffung von Parallelgesellschaften und Frontenverhärtung führt.

Terrorismus ist also ein soziales Phänomen und keine Krankheit. Daher kann bei der psychiatrischen Sichtweise des Menschen als bio-psycho-soziale Einheit auch nur eine Begegnung auf allen drei Ebenen zu Veränderung führen.

Fazit: Die Behandlung von Terroristen ist keine rein psychiatrische Angelegenheit, aber ohne die Psychiatrie wird es schwer. Und Wegsperren allein geht gar nicht. 